\title{
PROOF OF A CONJECTURE OF DOOB
}

\author{
J. S. HWANG AND D. C. RUNG
}

\begin{abstract}
Let $\mathscr{D}(\rho)$ be the class of all holomorphic functions $f$ in the unit disc $\Delta$ such that $f(0)=0$ and there exists an arc $\Upsilon_{f} \subseteq \partial \Delta$ with length $\left|\Upsilon_{f}\right|>\rho$ such that $\lim |f(z)|>1, z \rightarrow \tau \in \Upsilon_{f}$. In 1935, J. L. Doob asked, in essence, whether the Bloch norms $\left\{\|f\|=\sup _{z \in \Delta}\left|f^{\prime}(z)\right|\left(1-|z|^{2}\right)\right\}$ have a positive lower bound for the class $\mathscr{D}(\rho)$. We show that if $f \in \emptyset(\rho)$ there exists a $z_{f} \in \Delta$ such that
\end{abstract}

$$
\left|f^{\prime}\left(z_{f}\right)\right|\left(1-\left|z_{f}\right|^{2}\right)>\frac{2}{e} \frac{\sin (\pi-\rho / 2)}{(\pi-\rho / 2)} .
$$

1. Introduction. For fifty years the problem of determining precise estimates for the Bloch and Landau constants has been pursued actively. We describe the problems with the unit disc $\Delta$ in the complex plane as the domain for all functions which are assumed to be holomorphic unless otherwise specified. The Bloch number of a function $f$ is

$$
\begin{aligned}
& b(f)=\sup \left\{r \mid \text { there exists a subdomain } \Delta_{1} \subseteq \Delta \text { such that } f\right. \text { is } \\
& \text { univalent on } \Delta_{1} \text { and } f\left(\Delta_{1}\right) \text { contains a (Euclidean) disc } \\
& \text { of radius } r\},
\end{aligned}
$$

and the Landau number of $f$ is

$$
l(f)=\sup \{r \mid f(\Delta) \text { contains a (Euclidean) disc of radius } r\} .
$$

For a family of functions $\mathscr{F}$ the corresponding constants are

$$
b(\mathscr{F})=\inf b(f), \quad f \in \mathscr{F} ; \quad l(\mathscr{F})=\inf l(f), \quad f \in \mathscr{F} .
$$

Of course $b(\mathscr{F})<l(\mathscr{F})$. Classically the family $\mathscr{F}$ was taken to be either $\mathscr{B}$, the family of all $f$ with $\left|f^{\prime}(0)\right| \geqslant 1$, or $\mathcal{S}$, the family of all univalent $f$ with $\left|f^{\prime}(0)\right|>1$. The best estimates are

$$
\begin{aligned}
\sqrt{3} / 4 & =.433 \ldots<b(\mathscr{B})<.472<\cdots<.50<l(\mathscr{B}) \\
& <.544<\cdots<b(\mathcal{S})<.658 .
\end{aligned}
$$

The lower estimate for $b(\mathscr{B})$ is by Heins [12], improving Ahlfors' estimate [1] $b(\mathscr{B})>\sqrt{3} / 4$; the upper estimate for $b(\mathscr{B})$ is by Ahlfors and Grunsky [2];

Presented to the Society, March 7, 1978 under the title On two problems of Doob about the ranges of analytic functions and on April 15, 1978 under the title $A$ conjecture of Doob; received by the editors May 11, 1978 and, in revised form, September 11, 1978.

AMS (MOS) subject classifications (1970). Primary 30A42; Secondary 30A72.

Key words and phrases. Bloch, Landau constants, differential maximal principle.

() 1979 American Mathematical Society 0002-9939/79/0000-0309/\$02.00 
the corresponding estimates for $l(\mathscr{B})$ are by Pommerenke [15] improving Ahlfors [1] and Robinson as reported by Hayman [11]. For $b(\delta)$ the estimates are by Landau [13] and Robinson [16]. Other families of functions have been studied in this connection (see, for example, Hayman [10]).

In a series of papers begun in 1932, Doob [5] [9] considered certain boundary value problems as well as Bloch and Landau constants for a class of functions with a different normalization from $\mathscr{B}$. For any $0<\rho<2 \pi$, Doob defined $\mathscr{D}(\rho)$ as the family of all functions $f$ with $f(0)=0$ such that there exists an open arc $\Upsilon_{f}$ (depending on $f$ ) in $\partial \Delta$ with length $\left|\Upsilon_{f}\right|>\rho$ and such that $\lim _{z \rightarrow \tau}|f(z)| \geqslant 1$ for each $\tau \in \Upsilon_{f}$. In [7] Doob showed that $b(\mathscr{D}(\rho))$ is positive for each $0<\rho<2 \pi$ and also that there exists a positive constant $a(\rho)$ such that $f \in \mathscr{B}$ implies $a(\rho) f \in \mathscr{D}(\rho)$. More precisely he showed that $a(\rho) f \circ g \in \mathcal{D}(\rho)$ for some function $g,|g|<1, g(0)=0$, where $g$ depends on $f$. The reverse implication was thought by Doob to be false. If $L$ is a Möbius transformation of $\Delta$ onto $\Delta$, then it is clear that $b(f \circ L)=b(f), l(f \circ L)=$ $l(f)$, and $(f \circ L)^{\prime}(0)=\left(1-|L(0)|^{2}\right) f^{\prime}(L(0))$. Doob's question now becomes whether or not $f \in D(\rho)$ implies that there exists a positive constant $\sigma(\rho)$ independent of $f$ and a point $z_{f} \in \Delta$ such that $\left|f^{\prime}\left(z_{f}\right)\right|\left(1-\left|z_{f}\right|^{2}\right)>\sigma(\rho)$. If such a constant does exist, it follows that $(\sigma(\rho))^{-1}(f \circ L) \in \mathscr{B}$ for any $L$ such that $L(0)=z_{f}$. Professor Doob brought this question to our attention several months ago.

2. Main result. Defintion. For $\Upsilon \subseteq \partial \Delta$ let $\omega(z, \Upsilon, \Delta)$ denote the harmonic measure at $z$ of $\Upsilon$ relative to $\Delta$, and set

$$
S(\rho, \Upsilon)=\{z \in \Delta \mid \omega(z, \Upsilon, \Delta) \geqslant \rho / 2 \pi\}, \quad 0<\rho<2 \pi,
$$

and let

$$
\sigma(\rho)=\frac{2}{e} \frac{\sin (\pi-\rho / 2)}{(\pi-\rho / 2)} .
$$

Our proof is based upon a sharpened form of the Lehto-Virtanen differential two constant theorem [14] due to S. Dragosh and D. C. Rung [4]. Because this result is unpublished we give a simplified version of the result sufficient for the main result.

Theorem D-R. Let $f$ be a meromorphic function in D. For some fixed $S(\rho, \Upsilon)$ suppose that

(i) $\sup _{z \in S(\rho, r)}|f(z)|=M<\infty$;

(ii) for each $\tau \in \Upsilon$, $\varlimsup_{z \rightarrow \tau}|f(z)|<m<M$;

(iii) there exists a $q \in \partial S(\rho, \Upsilon) \cap \Delta$ at which $|f(q)|=M$.

Then

$$
\left|f^{\prime}(q)\right|\left(1-|q|^{2}\right) \geqslant e \sigma(\rho) M \log (M / m) .
$$


Proof. According to the two-constant theorem with $\omega(z)=$ $\omega(z, \Upsilon, S(\rho, \Upsilon))$ we have for $z \in S(\rho, \Upsilon),|f(z)|<M(m / M)^{\omega(z)}$, with equality occurring at $z=q$. The level line $|f(z)|=M$ is tangent to $\partial S(\rho, \Upsilon)$ at $z=q$ and so $\left|f^{\prime}(q)\right| \neq 0$. If $n$ is the inner normal to $\partial S(\rho, \Upsilon)$ at $q$ then

$$
\left.\frac{\partial|f(z)|}{\partial n}\right|_{z=q}<\left.\frac{\partial \omega(z)}{\partial n}\right|_{z=q} M \log \frac{m}{M} .
$$

It is easy to calculate that

$$
\left.\frac{\partial|f(z)|}{\partial n}\right|_{z=q}=-\left|f^{\prime}(q)\right| \text { and }\left.\frac{\partial \omega(z)}{\partial n}\right|_{z=q}=\frac{e \sigma(\rho)}{1-|q|^{2}} .
$$

If these are substituted in the above inequality the result is proved.

THEOREM 1. If $f \in\left(\rho_{0}\right), 0<\rho_{0}<2 \pi$, there exists at least one point $z_{f} \in$ $S\left(\rho_{0}, \Upsilon_{f}\right)$ at which $\left|f^{\prime}\left(z_{f}\right)\right|\left(1-\left|z_{f}\right|^{2}\right)>\sigma\left(\rho_{0}\right)$.

Proof. To the contrary suppose

$$
\left|f^{\prime}(z)\right|\left(1-|z|^{2}\right)<\sigma\left(\rho_{0}\right)
$$

for all $z \in S\left(\rho_{0}, \Upsilon_{f}\right)$ and consider the meromorphic function $g=1 / f$. For a subarc $\Upsilon^{*} \subseteq \Upsilon_{f}$ let $M\left(\Upsilon^{*}\right)=\sup |g(z)|, z \in S\left(\rho_{0}, \Upsilon^{*}\right)$. (We allow $M\left(\Upsilon^{*}\right)=\infty$ if $g$ has a pole in $S\left(\rho_{0}, \Upsilon^{*}\right)$.) Theorem D-R given above implies that at a point $q \in \Delta \cap \partial S\left(\rho_{0}, \Upsilon^{*}\right)$ at which $|g(q)|=M\left(\Upsilon^{*}\right)<\infty$ we have

$$
\operatorname{e\sigma }\left(\rho_{0}\right) M\left(\Upsilon^{*}\right) \log M\left(\Upsilon^{*}\right)<\left|g^{\prime}(q)\right|\left(1-|q|^{2}\right) .
$$

After a brief calculation, assumption (1.0) gives from (1.1) that

$$
M\left(\Upsilon^{*}\right) \log M\left(\Upsilon^{*}\right)<(1 / e)\left(M\left(\Upsilon^{*}\right)\right)^{2}
$$

or

$$
\left(M\left(\Upsilon^{*}\right)\right)^{-1} \log M\left(\Upsilon^{*}\right)<1 / e .
$$

Because the function $x^{-1} \log x$ has a maximum value of $1 / e$ which occurs when $x=e$, the inequality (1.2) says that $M\left(\Upsilon^{*}\right)$ lies in one of the intervals $[1, e)$ or $(e, \infty]$. Also, since $g$ is a continuous function, the possible values of $M\left(\Upsilon^{*}\right)$ form a connected set. Because there exist small arcs $\Upsilon^{*}$ for which $M\left(\Upsilon^{*}\right)<e$, we must have $M\left(\Upsilon^{*}\right)<e$ for each arc $\Upsilon^{*}$. But $M\left(\Upsilon_{f}\right)=\infty$ since $0 \in \partial S\left(\rho_{0}, \Upsilon_{f}\right)$ and $g(0)=\infty$. This contradiction proves the theorem.

The constant $\sigma(\rho)$ is best possible, at least in the limiting case $\sigma(\rho) \rightarrow 2 / e$ as $\rho \rightarrow 2 \pi$. The functions $f_{n}(z)=z^{n}$ all are in $\mathscr{D}(\rho)$ for all $0<\rho<2 \pi$. A brief calculation shows that $\left|f_{n}^{\prime}(z)\right|\left(1-|z|^{2}\right)$ has a maximum value of $2(n /(n$ $+1))[1-2 /(n+1)]^{(n-1) / 2}$ which tends to $2 / e$ as $n \rightarrow \infty$.

This theorem yields a new approach to upper bounds for $b(\mathscr{B})$, in that $f \in \mathscr{D}(\rho)$ implies that $b(\mathscr{B})<b(f)(\sigma(\rho))^{-1}$ and a similar inequality for $l(\mathscr{B})$. For example, 


$$
f_{n}(z)=\frac{e^{n((z+1) /(z-1))}-e^{-n}}{1-e^{n((z+1) /(z-1))} e^{-n}}
$$

is in $\mathscr{D}(\rho)$ for each $n=1,2, \ldots$, and any $0<\rho<2 \pi$. Because $f_{n}$ omits $-e^{-n}, l\left(f_{n}\right)=\left(1+e^{-n}\right) / 2$ and so $l(B)<(\sigma(\rho))^{-1}\left(\left(1+e^{-n}\right) / 2\right)$. Letting $\rho \rightarrow 2 \pi$ and $n \rightarrow \infty$ gives $l(\mathscr{B}) \leqslant e / 4 \simeq .679$. Whether better estimates can be obtained awaits further study.

\section{REFERENCES}

1. L. V. Ahlfors, An extension of Schwarz's lemma, Trans. Amer. Math. Soc. 43 (1938), 359-364.

2. L. V. Ahlfors and H. Grunsky, Über die Blochsche Konstante, Math. Z. 42 (1937), 671-673.

3. A. Bloch, Les théorèmes de M. Valiron sur les fonctions entières, et la théorie de l'uniformisation, Ann. Fac. Sci. Univ. Toulouse 3 (1925), 1-22.

4. S. Dragosh and D. C. Rung, Normal functions bounded on arcs and a proof of the Gross cluster-value theorem, Hiroshima Math. J. (to appear).

5. J. L. Doob, The boundary values of analytic functions, Trans. Amer. Math. Soc. 34 (1932), 153-170.

6. The boundary values of analytic functions. II, Trans. Amer. Math. Soc. 35 (1933), 718-751.

7. The ranges of analytic functions, Ann. of Math. 36 (1935), 117-126.

8. $\longrightarrow$ Cluster values of sequences of analytic functions, Sankhyā Ser. A 25 (1963), 137-148.

9. One sided cluster value theorems, Proc. London Math. Soc. 3 (1963), 461-470.

10. W. K. Hayman, Some applications of the transfinite diameter to the theory of functions, J. Analyse Math. 1 (1951), 155-179.

11. _ Research problems in function theory, Athlone Press, Univ. of London, London, 1967.

12. M. Heins, A class of conformal metrics, Bull. Amer. Math. Soc. 67 (1961), 475-478.

13. E. Landau, Über die Blochsche Konstante und zwei verwandte Weltkonstanten, Math. Z. 30 (1929), $608-634$.

14. O. Lehto and K. I. Virtanen, Boundary behavior and normal meromorphic functions, Acta Math. 97 (1957), 47-65.

15. C. Pommerenke, Bloch functions, J. London Math. Soc. 2 (1970), 689-695.

16. R. M. Robinson, The Bloch constant $\mathscr{A}$ for a schlicht function, Duke Math. J. 1 (1935), 535-540.

Department of Mathematics, McMasters University, Hamilton, Ontario, Canada L8S4K 1

Department of Mathematics, Pennsylvania State University, University Park, PennSYLVANIA 16802 (Current address of D. C. Rung)

Current address (J. S. Hwang): Institute of Mathematics, Academia Sinica, Tapei, Taiwan 